tive immunotherapy combination agents were mostly tested in checkpoint-inhibitor-naive patients. By way of comparison, Wilmington, Delaware-based Incyte's epacadostat, an IDO inhibitor tested in separate kidney, lung, bladder, and head and neck cancer trials in combination with Merck's PD-1 inhibitor Keytruda (pembrolizumab), delivered objective responses of 29-39\%, and Incyte shares leapt $17 \%$ following the data release at ASCO (J. Clin. Oncol. 35, annual meeting abstracts 4515, 9014, 4503, 6010, 2017). But Padmanee Sharma, a co-leader of the immunotherapy platform at the MD Anderson Cancer Center in Houston, who tracks the field closely, is not aware of any immunotherapy agent except CPI-444 that has publicly reported objective antitumor responses in PD-1 failures, although trials of such agents are ongoing. Bristol-Myers Squibb in Princeton, New Jersey, recently reported $16 \%$ objective responses for its Lag-3 inhibitor in PD-1 failures in melanoma (Nat. Biotechnol. 35, 487-488, 2017).

But the CPI-444 objective responses are few and this suggests problems. First, it's hard for drugs to outcompete the adenosine cloud for binding to the A2A receptor. "I'm not surprised that the responses have been somewhat limited, because I still think there's an issue about getting enough drug to the tumor," says Linden. One solution is to halt adenosine pro-
That's where the action is right now. Adenosine blockade, he's confident, is going to be an arrow in the immunotherapy quiver. the receptors that you care about very hard," says Arcus CEO Terry Rosen. Adds company president Juan Jaen, “I just don't think that the three repurposed CNS [central nervous system] drugs [from Corvus, Novartis in Basel, Switzerland, and AstraZeneca in Cambridge, UK; Table 1] are actually going to do that. At least not as fully as they could." Arcus' lead adenosine-targeted compound, the first designed specifically for cancer and scheduled to begin trials in October, targets both the $\mathrm{A} 2 \mathrm{~A}$ receptor, mainly found on $\mathrm{T}$ cells, and the $\mathrm{A} 2 \mathrm{~B}$ receptor, present on myeloid cells. The privately held company has raised $\$ 120$ million in two funding rounds.

Biomarkers such as CD73 in tumor biopsies - if validated - could prove valuable for patient selection, boosting drug response rates. Corvus is exploring the CD73 biomarker, as well as adenosine levels in blood, and a predictive gene expression panel.

Meanwhile, immunotherapy combinations are proliferating wildly. A recent review listed 123 such trials, all with a strong rationale (Cell 168, 707-723, 2017). "How to make sense of them?" asked University of California, Los Angeles, cancer researcher Antoni Ribas during an ASCO discussion session. His view is that the only combinations worth advancing are those that yield responses approaching $20 \%$ in patients who have failed anti-PD-1 or PD-L1 therapy, or in CD73, the enzyme that produces extracellular adenosine. Two companies have antiCD73 monoclonal antibodies in early trials (Table 1), with at least three more on the way. Combining anti-CD73 and anti-A2A receptor drugs, thus hitting adenosine production and signaling simultaneously, is an obvious approach.

Also, the current drugs are not ideal. Because they were developed for brain disease, the small molecules cross the bloodbrain barrier, a drawback in most cancer treatment. CPI-444 has shown good safety so far, but Arcus Biosciences in Hayward, California, has discovered small-molecule compounds with minimal brain penetration. That should mean more aggressive dosing. "You really want to be able to hit tumor types where such checkpoint inhibitors have been ineffective. "Anything that gets to $30 \%$, I'll jump out from my chair and start applauding," he said. Nothingnot IDO inhibitors, not agonist antibodies to costimulatory receptors like GITR and OX40, and not adenosine receptor blockers-yet meets this standard, including Corvus' drug. Industry attrition is already underway, with Genentech's July disclosure that it was abandoning both its IDO inhibitor and its OX40 antibody. Others are counting on patient selection. "Which tumors are responsive to different drugs, and different combinations?" asks Linden. "That's where the action is right now." Adenosine blockade, he's confident, "is going to be an arrow in the immunotherapy quiver."

Ken Garber Ann Arbor, Michigan

\section{Vertex CF data wow Wall Street}

On July 18, Vertex Pharmaceuticals announced dramatic results from triple-drug combinations that could potentially treat almost $90 \%$ of people with cystic fibrosis (CF). The three tripleagent combinations are the first investigational regimens to show a lung function benefit in individuals with both F508del mutations and minimal function mutations (F508del/min), a vast improvement in the proportion of $\mathrm{CF}$ patients amenable to treatment. Vertex's first CF drug Kalydeco (ivacaftor) is approved to treat mutations in the gene that gives rise to $\mathrm{CF}$, present in $6.7 \%$ of the global CF population, including those with certain residual function mutations (Nat. Biotechnol. 35, 606, 2017). Vertex's other approved CF drug, Orkambi (lumacaftor), counters defects caused by two copies of the F508del mutation present in about $50 \%$ of the patient population, although clinical improvements with this drug are modest. CF results from genetic alterations in the cystic fibrosis transmembrane conductance (CFTR) gene, which codes for a protein involved in ion transport across epithelial cell membranes. Kalydeco facilitates ion channel function on the cell surface, whereas Vertex's investigational agent tezacaftor improves the defective CFTR protein's positioning on the cell surface. Phase 3 studies with Kalydeco-tezacaftor combinations released in March 2017 were encouraging, but patients with minimal-function mutations, who have little-to-no functioning CFTR protein, failed to respond. In the latest studies, to target this group of patients, the Boston-based biotech added one of three experimental drugs that target minimal-function mutations (VX-445, VX-659 or VX-152) to the Kalydeco-tezacaftor combination. In the phase 2 studies with the triple combinations using VX-152 or VX-440 with Kalydeco-tezacaftor, the data revealed a $9.7 \%$ and $12.0 \%$ benefit, respectively, in lung function as measured by mean absolute improvements in percent predicted forced expiratory volume in one second $\left(p p F E V_{1}\right)$. A phase 1 study of $\mathrm{VX}-659$ in a triple combination showed a $9.6 \%$ lung function improvement. The safety and efficacy data are "clear and compelling, indicating significant benefit for people with CF from each of these three different triple combination regimens," Vertex executive vice president and chief medical officer Jeffrey Chodakewitz said in a press release.Vertex's stock jumped $21 \%$ on the news. Geoffrey Porges, biotech analyst with Leerink Research in New York, said that, "Within a year or two we cannot see any reason why $100 \%$ of the CF patient population who carry a F508del allele (roughly $87 \%$ of the total prevalent pool of patients) will not be on one of these medicines."

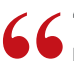

"I don't believe at all politics played a role in this. I wish it were so easy." John Crowley, CEO of Amicus Therapeutics in New Jersey, responds to comments that one day after he met with the president, the FDA reversed its decision to require further clinical trials for his company's drug Galafold, for treating a rare genetic disease that afflicts his daughter. 9 (STAT, 11 July 2017) 